\title{
Importancia de la evaluación de puesto de trabajo como herramienta de la evaluación y análisis ocupacional, quehacer cotidiano del Terapeuta Ocupacional, dentro de un contexto multivariado
}

Flor Esperanza Rodríguez Ferro*

PALABRAS CLAVES: Evaluación ocupacional, análisis ocupacional, métodos de evaluación de puesto de trabajo, ocupación, competencia laboral.

Desde la formación como Terapeutas Ocupacionales, el centro de actuar profesional, científico y académico se ha centrado en el estudio de la actividad como herramienta de intervención y más allá de esta el estudio de la ocupación; para el abordaje de las mismas se han identificado a través de la historia diversas técnicas de evaluación ocupacional, fundamentadas a diferencia de otras disciplinas en modelos propios de la Terapia Ocupacional. Pero en la práctica real, el abordaje de toda situación relacionada con la alteración del rol productivo del individuo, sin la generación o no de discapacidades, ha implicado la utilización de herramientas de evaluación ocupacional; las mismas tienen ventajas y desventajas, donde la clave de éxito radica en un adecuado procedimiento, ligado a la selección de acuerdo

\footnotetext{
* Terapeuta Ocupacional. Universidad Nacional de Colombia. Esp. ADM. Salud Ocupacional. Docente Rehabilitación Profesional, Ergonomía. Coordinadora práctica laboral, Facultad de Terapia Ocupacional. Escuela Colombiana de Rehabilitación.
} 
con las características y dificultades de los usuarios, el propósito del servicio, recursos disponibles, contexto real y tipos de trabajo, de ahí la importancia del papel conocedor que tiene el Terapeuta Ocupacional sobre la ocupación contextualizada en este artículo a los diferentes campos de competencia profesional dentro del contexto socio-económico y laboral del país; sector donde el T.O. ha adquirido posicionamiento y reconocimiento durante los últimos 15 años.

Dentro de la evaluación ocupacional, las herramientas utilizadas tienen un significado convencional: "son instrumentos utilizados en la realización de la tareas, o necesarios en la conducta de una ocupación" Su aplicación por lo tanto puede variar desde un contexto puramente preventivo como lo estipula la legislación actual en Colombia, hasta un contexto de intervención como es el caso de la Rehabilitación Profesional y de la Ergonomía; las herramientas utilizadas por lo tanto son el significado y el medio por el cual el Terapeutá Ocupacional logra participar en acciones de promoción del bienestar ocupacional, prevención de la enfermedad en un ámbito laboral, reintegro de población con discapacidad, así como la reorganización de procesos a nivel organizacional y productivo. La diferencia de su utilización va a depender de las "necesidades identificadas, de las que vayan surgiendo, más que de un plan predeterminado" (Vocational Evalutation and Work Adjusment Association, 1975, citado en GLARP, 1998).

El propósito último de la evaluación ocupacional es por lo tanto ayudar al usuario, independiente de la situación de estudio a obtener un autoentendimiento, autoconocimiento y la información necesaria para que se realice una mejor decisión vocacional, reubicación o inclusión socio-laboral, o mejoras al puesto de trabajo con base en situaciones de riesgo identificadas por el Terapeuta Ocupacional, así como establecimiento de relaciones de causalidad laboral, donde el T.O. relaciona el estudio de la ocupación y/o puesto de trabajo con las condiciones del ambiente y habilidades o características propias del individuo.

Como se aprecia entonces la evaluación ocupacional utiliza situaciones reales o simuladas con el fin de valorar habilidades personales para el trabajo. Existen diferentes clases de situaciones utilizadas como herramientas de evaluación: "evaluación en puestos de trabajo, en talleres de produccción, estaciones de trabajo simulado, multitaller, administración de pruebas, esayo de entrenamiento y seguimiento laboral. Estas situaciones usadas como herramientas de evaluación ocupacional identifican los hábitos de trabajo del cliente, intereses, habilidades y potencialida- 
des; también permiten revelar la motivación, nivel de maduración, autoimagen, resistencia física y emocional, desarrollo potencial, hàbilidad para relacionarse ... y muchas otras características que influyen en el empleo" (Brolin, 1982, citado por GLARP, 1998).

Con base en lo anterior el Terapeuta Ocupacional hace uso de herramientas, que incluyen el análisis ocupacional, información ocupacional; material audiovisual, técnicas para la recolección de información, registro y reporte (Chek List). Por último, se puede decir que la herramienta más importante y fundamental es la observación, en este caso aplicada aun puesto o situación de trabajo:

\section{Contextualización internacional sobre lo que es el análisis ocupacional}

El mismo parte de una breve contextualización sobre el mundo del trabajo y de la ocupación. La identificación de los contenidos de las ocupaciones se derivó de los intentos por lograr clasificaciones de los trabajos a fin de establecer diferentes niveles de remuneración. Los primeros antecedentes en la identificación de contenidos del trabajo derivaron en las categorías de clasificaciones de trabajadores para efectos de negociación colectiva. En esos años de comienzos del siglo XX, las diferenciaciones más descriptivas no pasaban de referirse a categorías como "trabajador", "empleado", "capataz", "supervisor", "gerente"; reflejando así, el estado de la organización del trabajo. Posteriormente las descripciones aparecieron muy ligadas a la lógica de los puestos de trabajo descritos; apegadas a la descripción exhaustiva pero también incluyendo un alto ingrediente jerárquico; diferenciando el trabajo de planta, del trabajo de oficina ý el trabajo de hacer, del trabajo de pensar ${ }^{1}$.

Con el tiempo las clasificaciones fueron adquiriendo complejidad; su creciente importancia en la negociación salarial ocasionó la intervención del estado para su definición. Cada vez más, nuevas ramas de producción fueron objeto de clasificación y las mayor cantidad de definiciones disponibles desembocó en el perfeccionamiento de técnicas de análisis de puestos de trabajo. Se diseñaron y establecieron un conjunto de métodos para ponderar diferentes factores de incidencia que

1 Jobert, Annette. "Las grillas de clasificación profesional, algunas referencias históricas". En Formación Profesional: Calificaciones y clasificaciones profesionales. Piette-Humanitas. Buenos Aires, 1990 
pretendían establecer la complejidad y profundidad de un puesto de trabajo de modo que se pudieran especificar las características educativas, habilidades, destrezas y aún, condiciones físicas de los candidatos. También, las técnicas de análisis de puestos se utilizaron para diseñar escalas salariales basadas en aspectos como la responsabilidad, esfuerzo físico, esfuerzo mental, ambiente de trabajo, etc.

Los cambios en los contenidos ocupacionales y en las formas de organización del trabajo; así como las nuevas exigencias en el desempeño competente de los trabajadores han delatado la obsolescencia de los métodos «científicos» de análisis de puestos. La alta especificación de tales análisis riñe con la flexibilidad requerida en el desempeño eficiente. La fragmentación de actividades propia del análisis de puestos no va con la polivalencia y mayor participación exigida. La tradicional diferenciación entre quien hace y quien decide se diluye en las nuevas formas de organización de equipos de trabajo autónomos y en la disminución de los niveles medios, típica de las estrategias de aplanamiento organizacional. En la actualidad se han perfeccionado metodologías para el análisis ocupacional que pretenden identificar contenidos ocupacionales y facilitar la descripción de las competencias requeridas para el desempeño en una ocupación. A partir de tal descripción se siguen sustentando muchas de las actividades de gestión de recursos humanos (selección, promoción, remuneración, capacitación, certificación, evaluación).

A continuación se citan algunas definiciones de análisis ocupacional de Cinterfor/ OIT, el INEM de España, la Secretaría de Trabajo y Previsión Social de México, el Servicio Nacional de Aprendizaje SENA y el American College Testing (ACT):

Cinterfor/OIT: Proceso de identificación a través de la observación, la entrevista y el estudio, de las actividades y requisitos del trabajador y los factores técnicos y ambientales de la ocupación. Comprende la identificación de las tareas de la ocupación y de las habilidades, conocimientos, aptitudes y responsabilidades que se requieren del trabajador para la ejecución satisfactoria de la ocupación, que permiten distinguirla de todas las demás².

La OIT en su glosario de términos ${ }^{3}$ define el análisis ocupacional como la "acción que consiste en identificar, por la observación y el estudio, las actividades y factores

${ }^{2}$ Agudelo, Santiago. Certificación ocupacional. Manual didáctico. Cinterfor/OIT. Montevideo, 1993.

${ }^{3}$ OIT. Formación profesional. Glosario de términos escogidos. Ginebra, 1993. 
técnicos que constituyen una ocupación. Este proceso comprende la descripción de las tareas que hay que cumplir, así como los conocimientos y calificaciones requeridos para desempeñarse con eficacia y éxito en una ocupación determinada".

Instituto Nacional de Empleo de España (INEM): El proceso de análisis ocupacional se centra en la revisión de diferentes fuentes (clasificación de ocupaciones, información económica sectorial, estudios de necesidades de formación) y se desarrolla en dos grandes fases: la primera es el establecimiento de la estructura ocupacional de la familia profesional y la segunda es la determinación de perfiles profesionales de las ocupaciones. Utiliza el método de análisis funcional y lo considera un instrumento superador del análisis de tareas. Considera a la ocupación una agrupación de actividades profesionales pertenecientes a diferentes puestos de trabajo con características comunes, cuyas tareas se realizan con normas, técnicas y medios semejantes, y responden a un mismo nivel de cualificación.

El perfil profesional, resultante en la segunda fase, es la descripción de competencias y capacidades requeridas para el desempeño de una ocupación, así como sus condiciones de desarrollo profesional. Esta compuesto por la declaración de la competencia general, la descripción de las unidades de competencia; la identificación de las realizaciones profesionales, la descripción y agrupamiento de las tareas y la especificación de los criterios de ejecución" ${ }^{4}$.

La Secretaría del Trabajo y Previsión Social de México define el análisis ocupacional como una «metodología enfocada a la obtención, ordenación y valoración de datos relativos a los puestos de trabajo, los factores técnicos y ambientales característicos en su desarrollo y las habilidades, conocimientos, responsabilidades y exigencias requeridas a los trabajadores para su mejor desempeño. Por ello, se recaba la información en los centros de trabajo, se clasifican en ocupaciones los puestos relacionados entre sí y se integran, una vez clasificados, en un catálogo ${ }^{5}$. El SENA define un concepto de "estudio ocupacional" como: "la recopilación sistemática, procesamiento y valoración de la información referente al contexto empresarial, económico, laboral, tecnológico y educativo de un sector ocupacional, a las funciones que desarrollan las empresas de ese sector para lograr su propósito, a las es-

4 INEM. Metodología para la ordenación de la formación profesional ocupacional. Madrid, 1995.

${ }^{5}$ Secretaría del Trabajo y Previsión Social. Catálogo Nacional de Ocupaciones. México, 1986. 
tructuras ocupacionales y a las competencias laborales asociadas a cada área ocupacional"6. El proceso que está adelantando esta institución en el ámbito de la formación basada en competencia laboral facilita la definición del estudio ocupacional asociado no solo a la identificación de las características del sector ocupacional, sino también, a la identificación de las funciones productivas y elaboración de normas de competencia laboral y titulaciones requeridas por el sector.

Para el American College Testing (ACT) es la "recolección sistemática y analítica de la información sobre las acciones que realizan los empleados en el desempeño de las tareas relacionadas con su empleo".

Esta organización desarrolló una metodología de análisis ocupacional en 1993 para identificar las competencias y destrezas comunes a través de todas las ocupaciones dentro de un entorno de trabajo ${ }^{7}$. Una vez obtenidos los comportamientos comunes, se pide a grupos de trabajadores que clasifiquen tales comportamientos en

${ }^{6}$ SENA. Guía para la elaboración de estudios ocupacionales. Dirección de empleo. Bogotá, 1998.

${ }^{7}$ ACT. Fundamentos básicos para el desarrollo de las competencias de trabajo. lowa City. 1998. En general, en este análisis de puestos de trabajo interesa saber:

A. Qué hace el trabajador, qué comprende:

- Actividades de orden físico

- Actividades de orden intelectual

B. Cómo lo hace, qué incluye:

- Métodos de trabajo

- Máquinas, utensilios, materiales, instrumentos de medida, equipo utilizado.

- Normas a seguir, instrucciones escritas o verbales que recibe.

- Valoraciones que hay que hacer.

- Decisiones que hay que tomar.

C. Por qué lo hace, este apartado debe responder a las preguntas siguientes:

- ¿Por qué realiza ese cometido?

- ¿Cuál es su objeto?

- ¿Cuáles son las relaciones existentes entre las operaciones del mismo cometido y los cometidos del puesto o de otros puestos?

D. Qué requiere la tarea, esto es analizar las dificultades que plantea el desarrollo de los objetivos de cada puesto en términos de requisitos físicos, de aptitud, conocimientos y capacidad.

En general, agrupados por factores, los elementos que no podemos olvidar al elaborar el análisis de puesto son:

- Normas recibidas y asistencia que se recibe o puede recibir en el puesto de trabajo

- Control ejercido sobre el puesto de trabajo

- Responsabilidad

- Consecuencias de una actuación defectuosa en el puesto

- Esfuerzo físico

- Esfuerzo mental

- Dificultad de las decisiones

(Continua) 
términos de la importancia que tienen para su ocupación y la frecuencia con que los practican. Ya clasificados y ponderados; los comportamientos dan una idea del tipo de competencia que debe fortalecerse en los trabajadores para mejorar transversalmente su empleabilidad. De este modo, los programas educativos y de formación pueden enfocar el desarrollo de las competencias transferibles logrando mayores efectos en el empleo de los trabajadores.

En Terapia Ocupacional los conceptos presentados anteriormente se consolidan en una teoría genera sobre lo que es análisis ocupacional, donde se considera que el mismo conlleva un diagnóstico tanto de la situación actual como la futura en lo referente a organigrama, puestos y estructuras organizativas

1. Definición de todos los puestos de trabajo que están en el momento actual en el organigrama de la Empresa:

Análisis de Puestos de Trabajo para el diagnóstico de necesidades formativas : Por su importancia y por su gran utilidad se especifica más adelante este tipo de análisis perteneciente al análisis ocupacional (Pereda, 1993).

Los datos extraídos del Análisis de Puestos de Trabajo que interesan en formación son:

- Normalización del desempeño y rendimiento en la tarea.

- Identificación de las diversas actividades que integran la tarea.

- Determinación del modo en el que deben realizarse estas actividades.

- Habilidades, conocimientos y aptitudes básicas para el desempeño.

Cuando se realiza con el objetivo de concretar las necesidades formativas, el análisis debe estar centrado en cuáles son las funciones que se requieren en cada pues-

(Continuación del llamado 7)

- Conocimientos profesionales teóricos y prácticos necesarios

- Capacidad profesional entendida como habilidad necesaria

- Nivel intelectual

- Requisitos aptitudinales

- Requisitos físicos

- Riesgos en el puesto 
to. Es decir, esta investigación debe ser más sintética que analítica, centrada más en información cualitativa que cuantitativa.

2. Análisis de las familias ocupacionales, análisis de los puestos y definición de los objetivos y funciones a desarrollar en cada categoría, para poder así analizar las carencias específicas de cada una.

3. Análisis de las futuras estructuras organizativas, en función de:

- Futuros cambios tecnológicos.

- Implantación de nuevas estructuras por demanda de la producción.

- Cambios estratégicos en la Dirección.

- Extinción de puestos de trabajo.

- Creación de otros.

- Planes de carrera y traslados.

Como puede apreciarse entonces el análisis ocupacional con uno de sus productos estrella como es la evaluación de puesto de trabajo, implica para el T.O. una actualización constante, así como el conocimiento de las tendencias actuales a nivel mundial en áreas tales como: la administración de personal, economía y tendencias en el empleo para personas con o sin discapacidad, todas estas enmarcadas dentro de un contexto multivariado de la prevención no solo en salud, si no en trabajo, así como el conocimiento de un contexto juridico-legal que enmarca la responsabilidad y compromiso por la utilización de diversas herramientas según un objetivo claro de intervención, fundamentado en la necesidad del cliente. Actualmente el sector laboral, campo de acción donde el T.O. ha logrado mayor posicionamiento implica para el mismo el manejo de tendencias actuales que buscan en el individuo el máximo desarrollo de su competencia o cualificación laboral/ocupacional, conllevando un desempeño óptimo y realizante; pero ¿qué es una calificación laboral?

Las unidades de competencia constituyen módulos con un claro significado y valor en el trabajo. La agrupación de diferentes unidades en grupos con una clara configuración ocupacional del sector analizado y cori un nivel de competencia definido, va configurando las calificaciones laborales.

Las calificaciones no son nombres de puestos de trabajo. Son conjuntos de competencias que pueden servir como referente para el desempeño de los puestos de 
trabajo en la organización y también para la conformación de programas de formación. Cada puesto de trabajo tendrá claramente especificadas las unidades de competencia que deben ser certificadas para su ejercicio competente. Una calificación laboral puede tener unidades aplicables a más de un puesto de trabajo, así se empieza a facilitar la movilidad laboral.

Las calificaciones laborales son un conjunto de unidades de competencia integradas en el ámbito de una función productiva.

Para el CONOCER ${ }^{8}$ existe, en términos generales, una cierta similitud entre el concepto de calificación y el de profesión en cuanto esta contiene una gama amplia de posibilidades de desempeño en diferentes puestos.

Resumiendo, una calificación laboral, en un nivel de desempeño especificado; está constituida por varias unidades de competencia. Las unidades de competencia están conformadas por elementos de competencia y estos a su vez se especifican en criterios de desempeño, rango de aplicación, evidencias de conocimiento y evidencias de desempeño.

El concepto de calificación, utilizado entre otros, en el sistema mexicano, puede asemejarse al de titulación, empleado en el sistema del Reino Unido y recogido por el SENA bajo la siguiente definición: "Las titulaciones son grupos de normas de competencia que, en conjunto, establecen los niveles estándar de desempeño laboral para áreas ocupacionales específicas" 9 .

${ }^{8}$ CONOCER. Análisis ocupacional y funcional del trabajo. Op. cit.

${ }^{9}$ SENA. Guía para la elaboración de estudios ocupacionales. Dirección de Empleo. 1998.

\begin{tabular}{|l|l|}
\hline \multicolumn{1}{|c|}{ MÉTODO } & \multicolumn{1}{c|}{ AUTOR } \\
\hline Regie Renault. & Régie Nationale des Usines Renault \\
\hline Método LEST & $\begin{array}{l}\text { Laboratorio de Economía y Sociología del Traba- } \\
\text { jo de Aix-en- Provence }\end{array}$ \\
\hline Método Mapfre & Fundación Mapfre \\
\hline $\begin{array}{l}\text { Modelo Sueco para Adaptación de Puestos de Tra- } \\
\text { bajo }\end{array}$ & $\begin{array}{l}\text { Inger Rundstrom } \\
\text { Instituto de Empleo de Suecia }\end{array}$ \\
\hline
\end{tabular}


Para el SENA toda titulación agrupa competencias de áreas ocupacionales afines, representa desempeños significativos dentro de un área ocupacional y por supuesto, no es el nombre de un cargo o puesto de trabajo; cabe la posibilidad de que se identifiquen varias titulaciones dentro de una mismá área.

La guía del SENA ilustra varios ejemplos de titulaciones:

"Capacitación y desarrollo de personal".

"Producción de materiales impresos".

"Fabricación de productos metalmecánicos".

"Operación de plantas de agua potable".

(Continuación del llamado9).

\begin{tabular}{|c|c|}
\hline - . $\quad$ - MÉTODO & AUTOR \\
\hline Método Ertomis & Hans-Martin Schian, Arzt Fur Arbeits-Medizin \\
\hline $\begin{array}{l}\text { Método de Adaptación de puestos de trabajo para } \\
\text { usuarios en silla de ruedas }\end{array}$ & Page Álvaro. Instituto de Biomecánica de Valencia \\
\hline $\begin{array}{l}\text { Método del Diseño Ergonómico de puestos de tra- } \\
\text { bajo para personas con discapacidad física }\end{array}$ & $\begin{array}{l}\text { Mondelo Pedro } \\
\text { Universidad Politécnica de Cataluña (Barcelona) }\end{array}$ \\
\hline Método de perfiles & $\begin{array}{l}\text { Zurimendi Miguel Martin. } \\
\text { Lantegi Bataúk (Viscaya) }\end{array}$ \\
\hline $\begin{array}{l}\text { Método Binomio Ergonomia-Tecnología en el } \\
\text { empleo de personas con Discapacidad }\end{array}$ & $\begin{array}{l}\text { Garcia Camino Mercedez. } \\
\text { Grupo ATED, Madrid }\end{array}$ \\
\hline $\begin{array}{l}\text { Método de la Asociación de Prevención de Acci- } \\
\text { dentes } \\
\text { (A.P.A.) }\end{array}$ & $\begin{array}{l}\text { Instituto de Biomecánica de Valencia } \\
\text { Programa ERGO IBV }\end{array}$ \\
\hline \multicolumn{2}{|l|}{$\begin{array}{l}\text { Método Ovako Working Posture Analysis Sistem } \\
\text { (OWAS) }\end{array}$} \\
\hline Método NIOSH & $\begin{array}{l}\text { National Institute of Occupational Safety and } \\
\text { Health }\end{array}$ \\
\hline Job Analysis & $\begin{array}{l}\text { Division of Occupational Analysis } \\
\text { U.S Employement Service. } \\
\text { U.S. Department of Labor. }\end{array}$ \\
\hline
\end{tabular}

Fuente: Consolidado ên estüdio "MÉTODOS DE EVALUACIÓN DE PUESTO DE TRABAJO, UN ANÁLISIS A LA LUZ DE TERAPIA OCUPACIONAL, CONTEXTUALIZACIÓNMUNDIAL Elaborado por: Laserna, Rosario; Rodriguez, Esperanza, Bogotá, octubre de 2000. 
Vótese la alta afinidad de.los nombres de las titulaciones con procesos típicos de os análisis funcionales y su alejamiento de los tradicionales nombres de puestos de rabajo. Por lo tanto, el estudio de lo que es un puesto de trabajo, conlleva al aborlaje de métodos existentes en la actualidad a nivel mundial, orientadoś según la recesidad identificada a áreas tales como: la ergonomía, personas con discapacidad, elección de personal, procesos de reconversión laboral, determinación de ausalidad y reintegro o inclusión sociolaboral; de los mismos se enuncian a contiuuación algunos, pero su caracterización será presentada en un articulo posterior, ınalizados desde la perspectiva de Terapia Ocupacional, estos son estudiados en :I proceso de formación y en el actuar cotidiano evidencian la fortáleza del profeional en el área del análisis y evaluación ocupacional según el contexto de lesempeño.

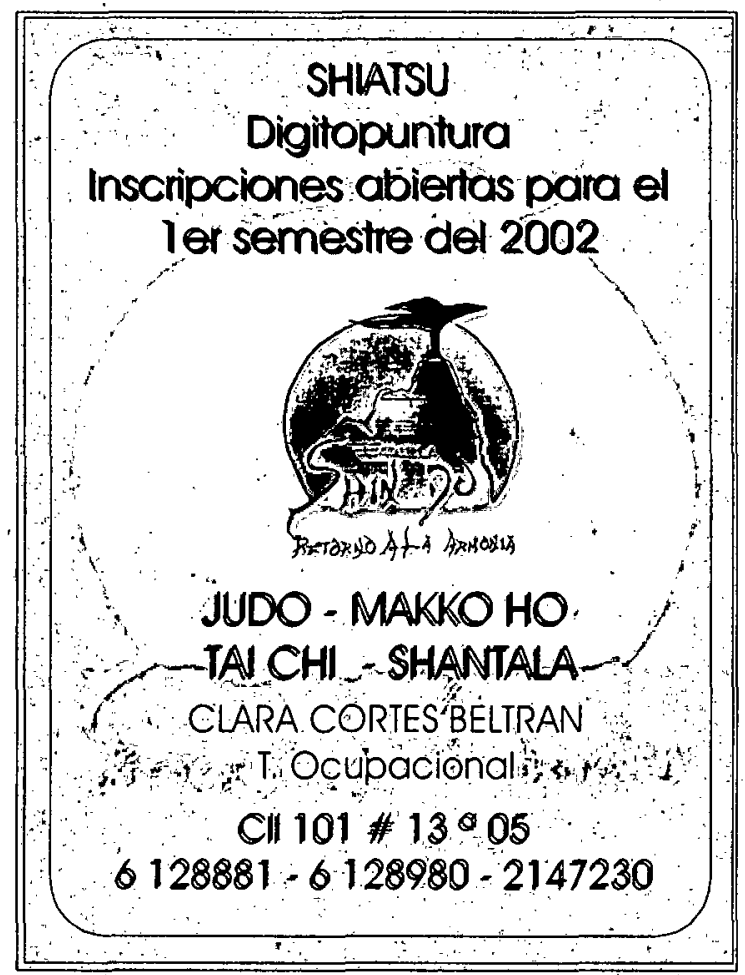

